\title{
One-pot oximation-Beckmann rearrangement of ketones and aldehydes to amides of industrial interest: Acetanilide, caprolactam and acetaminophen
}

\author{
Fabio Aricò ${ }^{\mathrm{b}, \mathrm{c}}$, Giuseppe Quartarone ${ }^{\mathrm{a}, \mathrm{b}}$, Elia Rancan ${ }^{\mathrm{a}, \mathrm{b}}$, Lucio Ronchin ${ }^{\mathrm{a}, \mathrm{b}, *}$, Pietro Tundo ${ }^{\mathrm{b}, \mathrm{c}}$, Andrea Vavasori ${ }^{\mathrm{a}, \mathrm{b}}$ \\ a Department of Molecular Science and Nanosystems, Ca' Foscari University, 2137 Dorsoduro, 30123 Venice, Italy \\ b Green Chemistry Group, 2137 Dorsoduro, 30123 Venice, Italy \\ c Department of Environmental Science, Informatics and Statistics, Ca' Foscari University, 2137 Dorsoduro, 30123 Venice, Italy
}

\section{A R T I C L E I N F O}

\section{Article history:}

Received 24 December 2013

Received in revised form 3 February 2014

Accepted 4 February 2014

Available online 12 February 2014

\section{Keywords:}

Amides

Oximation

Beckmann rearrangement

Trifluoroacetic acid

Ketones

\begin{abstract}
A B S T R A C T
High yielding one-pot oximation-Beckmann rearrangement of ketones to amides in ktrifluoroacetic acid has been conducted on several ketones and aldehydes. The substrate reactivity showed to depend on both oximation and Beckmann rearrangement reaction rate. In this synthetic procedure, trifluoroacetic acid acts as solvent, acid catalyst and organocatalyst and can be easily recycled.
\end{abstract}

(C) 2014 Elsevier B.V. All rights reserved.

\section{Introduction}

Amides are important building blocks in organic and material chemistry as they are widely employed not only in plastic, rubber, paper and color industry (crayons, pencils and inks), but also in water and sewage treatment[1-3]. Furthermore, numerous pharmaceutical molecules incorporate amides as core unit; $\mathrm{N}$-acetyl-4-aminophenol and local anesthetic lidocaine and dibucaine are just few examples [1-4]. Thus, considering their importance as intermediate in the industry and as precursor in drug formulation, the development of simpler and more economical process for amide synthesis has been of great interest over the last twenty years [5].

Among the most commonly used synthetic approaches for these compounds many involve the reaction of amine with anhydrides, acyl chlorides or, in some cases, with the acid itself [1]. This latter approach results in the complete conversion of the substrate only when the water, formed during the reaction, is continuously removed [1].

Amides can also be synthesized by a two step reaction: oximation of ketones, a quite facile reaction generally carried out with hydroxylamine hydrochloride or sulfate in an aqueous or water-ethanol solution, followed by Beckmann rearrangement in mineral acids [4-10]. The first step proceeds in the presence of a base to allow the formation

\footnotetext{
* Corresponding author at: Department of Molecular Science and Nanosystems, Ca' Foscari University, 2137 Dorsoduro, 30123 Venice, Italy. Tel.: + 390412348626. E-mail address: ronchin@unive.it (L. Ronchin).
}

of the free hydroxylamine that then attacks the carbonyl group of the ketone.

On the other hand, Beckmann rearrangement is generally carried out in mineral acid i.e. $\mathrm{H}_{2} \mathrm{SO}_{4}$ or oleum [5-11], for which safety and/or disposal problems must be taken into account especially in the industrial practice [12-17]. Furthermore, the resulting amides are protonated, thus, dilution with water or neutralization of the acid (typically with aqueous ammonia) is required to recover the pure product [12-17]. A commodity produced according to this synthetic approach is caprolactam, the monomer of nylon [12-17]. Nowadays the oximationrearrangement sequence in oleum is superseded by the EnichemSumitomo processes consisting of liquid phase ammoximation [13] followed by a gas phase Beckmann rearrangement [14-17].

Another interesting approach to amide synthesis uses ionic liquids in combination with Lewis acids. This procedure results in a high yielding Beckmann rearrangement for some activated oximes $[18,19]$. The process, however, requires a preliminary oximation stage and a tedious work-up for both oximation and Beckmann rearrangement.

Recently, examples of direct oximation-Beckmann rearrangement of cyclohexanone to $\varepsilon$-caprolactam has also been reported in liquid phase reaction starting from cyclohexanone, ammonia and air in the presence of bifunctional catalysts [20,21]. Similar results have been claimed by Uhde/Inventa-Fischer in the heterogeneously catalyzed liquid phase ammoximation-Beckmann rearrangement of cyclohexanone to caprolactam [22]. These processes are complex one-pot three-step reactions: ammonia oxidation to hydroxylamine, oximation of cyclohexanone and Beckmann rearrangement of the cyclohexanone oxime. 
However, the final product can be isolated only in moderate yield (20$50 \%$ ) and the procedure has not been investigated on different ketones.

Several metal-catalyzed one-pot syntheses of amides from aldehyde have also been reported although these processes require either long reaction time, high temperature or toxic solvents $[23,24]$. In additions, these reactions do not occur with ketones which limit their synthetic interest.

The use of trifluoroacetic acid (TFA) as catalyst in the presence of $\mathrm{CH}_{2} \mathrm{Cl}_{2}$ as solvent was firstly reported by Cossy and co-workers in the Beckmann rearrangement of oxime carbonate [25]. In this synthetic approach the key step is the formation of the oxime carbonate being more activated than the naked oxime in the Beckmann rearrangement due to the electron withdrawing effect of the carbonate group.

TFA has been also employed as catalyst for the Beckmann rearrangement of cyclohexanone oxime to $\varepsilon$-caprolactam [26-28]. In particular, a $\mathrm{TFA} / \mathrm{CH}_{3} \mathrm{CN}$ mixture was used for a practical and high yielding synthesis of amides [26-28]. The proposed reaction mechanism envisages the formation of the oxime ester of the trifluoroacetic acid, which, after rearrangement, forms a trifluoroacetyl amide. This compound is the key intermediate of the trifluoroacetylation process of the oxime as it continuously reforms sustaining the catalytic cycle (Scheme 1).

In our previous work we outlined that the mechanism of the Beckmann rearrangement in TFA occurs via esterification of the oxime also for the acetophenone oxime and for the 4-hydroxyacetophenone oxime [29].

Recently, Luo and co-workers reported the Beckmann rearrangement of cyclohexanone oxime to caprolactam in $\mathrm{TFA} / \mathrm{CH}_{3} \mathrm{CN}$ optimizing the caprolactam yield by using conditions similar to those employed in our previous papers [26-30]. The same authors reported an oximationBeckmann rearrangement of cyclohexanone to caprolactam using a $\mathrm{TFA} / \mathrm{CH}_{3} \mathrm{CN}$ system [31]. This synthetic procedure, although interesting, focuses only on one substrate i.e. caprolactam without discussing the general applicability of the system.

In this work, following our previous investigation on the Beckmann rearrangement of ketoximes, we account on a general approach for the synthesis of amides starting from ketones or aldehydes via a one-pot oximation-Beckmann rearrangement. Hydroxylamine is used in industrial processes as oximating agent after neutralization of its sulfate salt being hydroxylamine itself an unstable reagent [1-4]. Here we used hydroxylamine hydrochloride, which is a stable and soluble salt, as oximation agent and TFA as catalyst and solvent. The reaction is of general application and results in the high yielding preparation of amides. The use of TFA as catalyst and solvent renders the processes both sustainable and highly efficient. In fact, the reaction does not require any work-up operations as TFA can be removed by low temperature vacuum distillation and recycled. Furthermore, due to the TFA low protonation ability, the resulting amides can be easily recovered as pure compounds.

\section{Experimental}

\subsection{Materials}

All the solvent and products were employed as received without further purification. Acetophenone $\geq 98 \%$, acetone $\geq 99.9 \%$, 2-hydroxyacetophenone $\geq 98 \%$, 4-methylacetophenone $\geq 95 \%$, 2-methylacetophenone $\geq 98 \%, 2,4,6$ trimethylacetophenone $\geq 98 \%$, 4-bromo acetophenone $98 \%$, 2-bromo acetophenone $98 \%$, propiophenone $99 \%$, butyrophenone $\geq 99 \%$, 2,2-dimethylpropiophenone $98 \%$, isobutyrophenone $97 \%$, benzophenone $\geq 99 \%$, 4-phenyl-2-butanone $98 \%$, ethyl benzoylacetate 97\%, 4-nitrobenzaldehyde 98\%, 4-isopropylbenzaldehyde 98\%, 2-hydroxy benzaldehyde $\geq 98 \%$, hexanal $98 \%$, trifluoroacetic acid $99 \%$, and hydroxylamine hydrochloride $99 \%$ were all Aldrich products; 4-hydroxiacethophenone $\geq 98 \%$ (HPLC) was a HPLC grade Fluka product. Cycloexanone $99.8 \%$ was an ACROS reagent. Benzaldehyde 99\% was a Carlo Erba reagent. Deuterated chloroform and deuterated DMSO-d6 were EurisoTop products.

\subsection{Instruments and analysis}

Reaction products were analyzed by Gas Chromatography (GC) and Gas Chromatography coupled to Mass Spectroscopy (GC-MS), using an Agilent model 5975C interfaced with a GC Agilent model 7890 a HP5 capillary column (300 $\mu \mathrm{m}$ i.d. $30 \mathrm{~m}$ long, 95\% methyl, 5\% phenyl silicone phase).

The samples were also checked by a high performance liquid chromatography (HPLC). The instrument employed was a Perkin Elmer binary LC pump 250 with phenomenex Luna, $5 \mu \mathrm{m} \mathrm{C18} 100 \AA$, LC column $30 \mathrm{~mm} \times 4.6 \mathrm{~mm}$ (detector: Perkin Elmer LC 235 C Diode Array), wavelengths: $255 \mathrm{~nm}$ and $220 \mathrm{~nm}$; eluent: water-acetonitrile with a concentration gradient $60 \%$ water ( $9 \mathrm{~min}), 50 \%$ water $(5 \mathrm{~min})$ and $30 \%$ water (1 $\mathrm{min})$.

The ${ }^{1} \mathrm{H}$ Nuclear Magnetic Resonance (NMR) spectra were recorded on a Bruker AC 200 spectrometer operating at $200.13 \mathrm{MHz}$, and the sample temperature was maintained at $298 \mathrm{~K}$. All the chemical shifts were referred to internal tetramethylsilane.

\subsection{Ketones reactivity}

All the reactions were carried out in a well stirred pressurized glass reactor thermostated at $70{ }^{\circ} \mathrm{C}$ temperature and containing weighed samples of the solvent and reagents.

In a typical experiment a glass reactor equipped with magnetic bar was charge with $1.5 \mathrm{mmol}$ of the selected ketone or aldehyde, $4.4 \mathrm{mmol}$ of hydroxylamine hydrochloride and $22 \mathrm{mmol}$ of trifluoroacetic acid under inert atmosphere of nitrogen. The reaction time was computed after the heating fluid starts to circulate in the<smiles>O=C(ON=C1CCCCC1(O)C(=O)O)OC(F)(F)F</smiles><smiles>C/C(=N/OC(=O)C(F)(F)F)ON=C1CCCCC1</smiles> 
reactor jacket. The reaction mixture was heated at $70{ }^{\circ} \mathrm{C}$ for $16 \mathrm{~h}$, and then cooled down.

Products were isolated after distillation of the TFA (at $40{ }^{\circ} \mathrm{C}$ and $250 \mathrm{~Pa}$ of pressure) and in a rotary evaporator, washed with cold water and recrystallized from hexane or light petroleum ether.

In selected cases, the product was purified by column chromatography employing dichloromethane/diethyl ether 9:1 as elution mixture. In all the conducted experiments, analysis of the isolated products was consistent to the data reported in the literature (see Supplementary materials).

\section{Results and discussion}

Table 1 reports the results achieved for the one-pot oximationBeckman rearrangement reaction on some selected aromatic and aliphatic ketones (Scheme 2). In all the experiments the amide resulted the main product formed with selectivity up to $99 \%$; the formation of oxime intermediate has also been sometime observed.

It is evident that, being a multistep process, the outcome of the reaction depends on the reactivity of the substrate toward both oximation reaction and Beckmann rearrangement. Consequently, acetophenone, acetone and cyclohexanone (entries 1-4, Table 1), whose structures allow an easy oximation, are converted into amides in high yield (70$90 \%$ ). This result also implies a facile Beckmann rearrangement of the corresponding oximes.

It is interesting to observe that the highest conversion was obtained when cyclohexanone was used as substrate, suggesting an easy oximation stage. However, results collected indicate that the rearrangement was only partially completed as demonstrated by the presence of cyclohexanone oxime (11\%). The presence of oxime depends on the relative rate of reaction of the two reaction steps, but also on the solvent, acidity and steric hindrance phenomena [6-10].

The reactivity of mono-substituted aryl ketones was then investigated (entries 4-8, Table 1). It is noteworthy that in our reaction conditions, $\mathrm{N}$-acetyl-4-aminophenol, the widely used drug acetaminophen, can be easily synthesized starting from 4-hydroxy-acetophenone (entry 4, Table 1). In this case, the pure product can be isolated by recrystallization after TFA removal by a rotary evaporator.

On the other hand, when the substrate incorporates a sterically hindered carbonyl group, the oximation resulted more difficult (entries 6 and 8, Table 1) [6,18]. This result also explains the different reactivities of 4-methyl acetophenone (entry 5) and 2-methyl acetophenone (entry 6 ) as the conversion of the latter substrate in the related amide is only moderate (entries 5 and 6 respectively, Table 1).

An analogous trend was also observed for the 4- and 2bromoacetophenone (entries 7 and 8 , Table 1 ) as the ortho substituted substrates resulted less reactive than the para ones. It is noteworthy that when 2-substituted acetophenones were employed as substrates the formation of oximes intermediate was never observed. This is in agreement with Beckmann rearrangement being easier with 2- or 6substituted aryl ketones than with unsubstituted or 4-substituted ones [10,32]. This result might also suggest that, when hindered aryl ketones are employed, the nucleophilic substitution is the determining step of the reaction instead of the Beckmann rearrangement of the ketoxime.

Linear alkyl substrates methyl, ethyl and $n$-propyl phenylketones (entries 1, 9 and 10) have also been investigated, showing a conversion decreasing with the increasing of the aliphatic chain length, although the related amide is, in any case, the main product observed (entries $1,9,10$, Table 1 ).

The presence of a branched substituent, i.e. an isopropyl- or tertbutyl-moiety, influences the reaction conversion resulting in a nonselective formation of anilides (entries 11 and 12, Table 1). This is due to the competitive formation of the sin and anti-ketoximes that can be ascribed to the similar steric hindrance of phenyl or branched aliphatic groups [33]. In fact, when tert-butyl phenylketone (entry 12 ,
Table 1

Oximation-Beckmann rearrangement in trifluoroacetic acid of selected ketones. Run conditions: reaction time $=16 \mathrm{~h}$, substrate $=2 \mathrm{mmol}, \mathrm{T}=70{ }^{\circ} \mathrm{C}$, and substrate/ $\mathrm{NH}_{2} \mathrm{OH} \cdot \mathrm{HCl} / \mathrm{CF}_{3} \mathrm{COOH}=1 / 3 / 15$

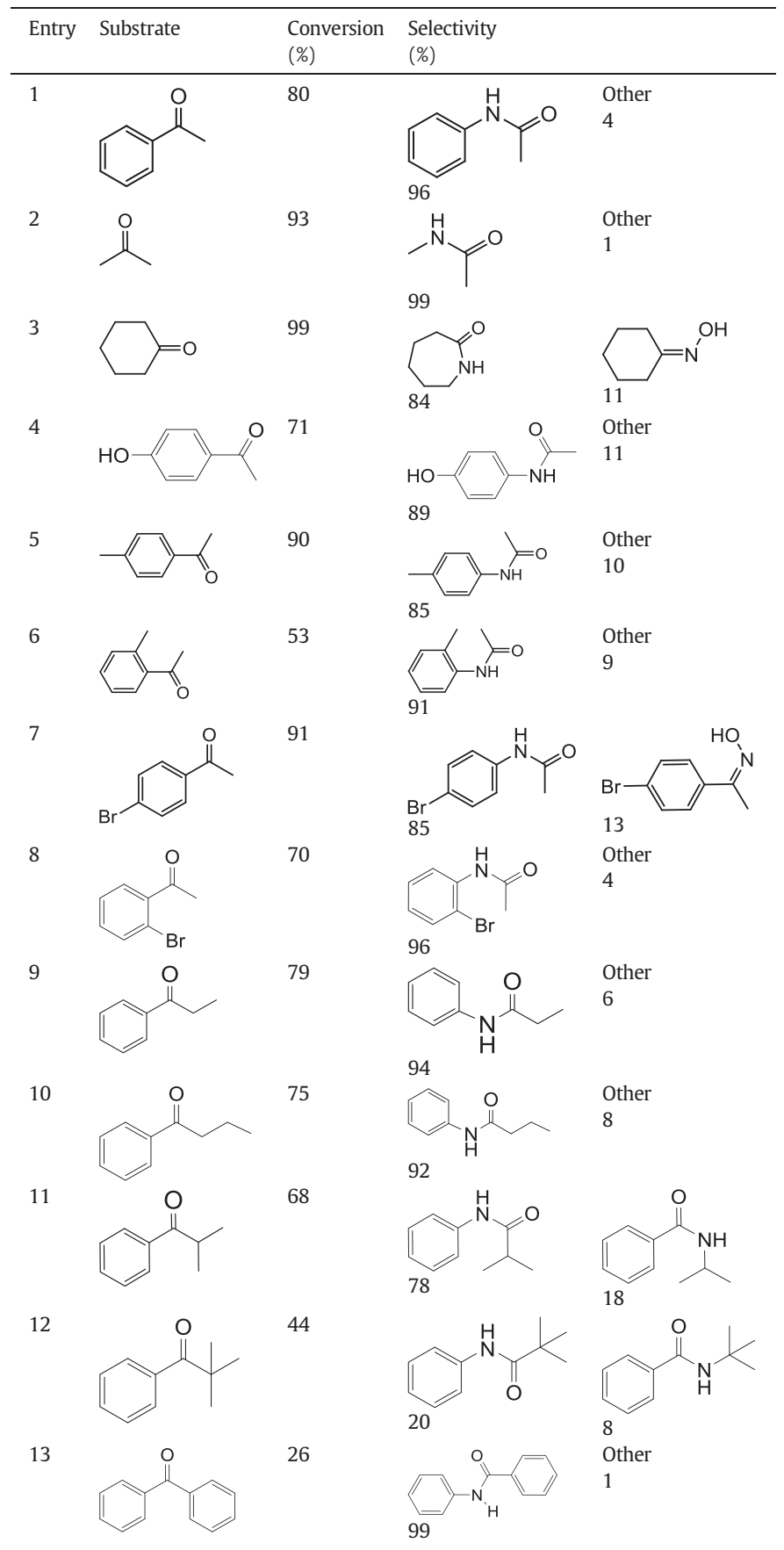

Table 1) was used as substrate the anilide/benzamide ratio detected was bigger than the one observed in the reaction involving isopropylphenylketone (entry 11 , Table 1 ) (78/18 and 20/8, respectively). This is an obvious consequence of the relative rate of formation of the sin and anti-ketoxime, which is determined by the steric hindrance of the alkyl group.

Finally, the extremely hindered benzophenone (entry 13, Table 1 ) is only scarcely converted into the $N$-phenylbenzamide although with an almost total selectivity to the rearranged amide. Most probably the low conversion can be ascribed to a less efficient oximation reaction due to the steric hindrance of the phenyl groups, while the high 
<smiles>[R]C([R])=O</smiles><smiles>N[C@H](O)[C@@H](O)Cl</smiles><smiles>[R]C([R])=NO</smiles><smiles>[R]NC([R])=O</smiles>

Scheme 2. Oximation-Beckmann rearrangement of ketones to amides.

selectivity can be ascribed to the stability of the starting ketone, which does not give side reactions.

In order to investigate the eventual limits of this synthetic procedure, the reactivity of complex ketones, incorporating different functional groups, has also been investigated (Table 2).

In particular, when 4-phenylbutan-2-one (entry 1, Table 2) was used as substrate, the expected amide formed with $70 \%$ selectivity. A significant amount of 4-phenylbutan-2-one oxime was also observed (25\%). This is in agreement with the results reported in Table 1 (entries 11-13) where the sterically hindered phenylalkyl moiety slowed down the rearrangement rate, diminishing the amide selectivity.

It should be noted that, in this synthetic procedure, the formation of amide might be affected by the $\mathrm{NH}_{2} \mathrm{OH}$ nucleophilic attack to the carbonyl group of the ketone, which is influenced by the steric hindrance of the substituents on the phenyl ring. In fact, in our reaction conditions, 1-mesytilethanone undergoes only deacylation and substitution reaction. Most probably the methyl groups in 2- and 6-positions hamper the nucleophilic substitution of the hydroxylamine to give the corresponding oxime so that the molecule is subjected to deacylation and substitution and the Beckmann rearrangement is completely suppressed. Such behavior agrees with the reactivity of the mesitoic acid toward esterification, which occurs only in the presence of very strong acid and severe conditions [34].

The reactivity of the 2-hydroxyacetophenone oxime (entry 3, Table 2) showed a low conversion and the main product was not the expected 2'-hydroxyacetanilide but the heterocyclic compound methyl benzoxazole, which is a useful intermediate employed in fine chemical production [4].

The one-pot oximation-Beckmann rearrangement reaction has also been tested on the ethyl benzoylacetate. This $\beta$-ketoester (entry 4 , Table 2) leads to a complex mixture of products, due to a non-selective nucleophilic attack $\left(\mathrm{NH}_{2} \mathrm{OH}\right)$ and to a competition among rearrangement and fragmentation reactions. The two major products formed were acetanilide and benzonitrile, but several products of fragmentation rearrangement and condensation have been also observed.

The reactivity of selected aldehydes in this reaction condition has also been investigated (Table 3). As expected aldehydes resulted more reactive than ketones giving generally a higher conversion (see for comparison Table 1). In fact, benzaldehyde was easily converted into benzamide in high yield; a small amount of benzonitrile was also detected [35]. A similar reactivity was observed for 4-nitrobenzaldehyde (entry 2), which formed 4-nitrobenzamide in high yields. 4-Isopropyl benzaldehyde (entries 3 ) gave almost quantitative conversion and high selectivity to the corresponding benzonitrile. Analogously, 2hydroxy benzaldehyde (entry 4) was mainly converted into benzonitrile together with a negligible amount of benzamide (only traces were observed at the GC-MS analysis).

The lower aldehyde conversion observed in this case (85\%) can be ascribed to the steric hindrance of the ortho hydroxyl group, which lowers the rate of the nucleophilic attack of the hydroxylamine as already pointed out for ketones (see Table 1 ).

Finally, hexanal (entry 5) gave a complex mixture of oxidation condensation and decomposition products, probably due to fast acid catalyzed unwanted reactions.

\section{Conclusions}

Herein we report on a one-pot oximation-Beckmann rearrangement of ketones and aldehyde as a novel synthetic pathway to achieve amides of industrial interest. The reaction has been conducted on several ketones resulting in a highly selective synthesis of amide, i.e. $\mathrm{N}$-acetyl-4aminophenol. The conversion of ketones into the final amide is strictly related to the reactivity of the substrate with respect to the two different steps of the reaction. When the ketone undergoes easy oximation then the Beckmann rearrangement is the determining step of the reaction. Similarly, if the steric hindrance of the substituent hampers the

Table 2

Reactivity of complex ketones. Run conditions: reaction time $=16 \mathrm{~h}$, substrate $=2 \mathrm{mmol}, \mathrm{T}=70{ }^{\circ} \mathrm{C}$, and substrate $/ \mathrm{NH}_{2} \mathrm{OH} \cdot \mathrm{HCl} / \mathrm{CF}_{3} \mathrm{COOH}=1 / 3 / 15$.

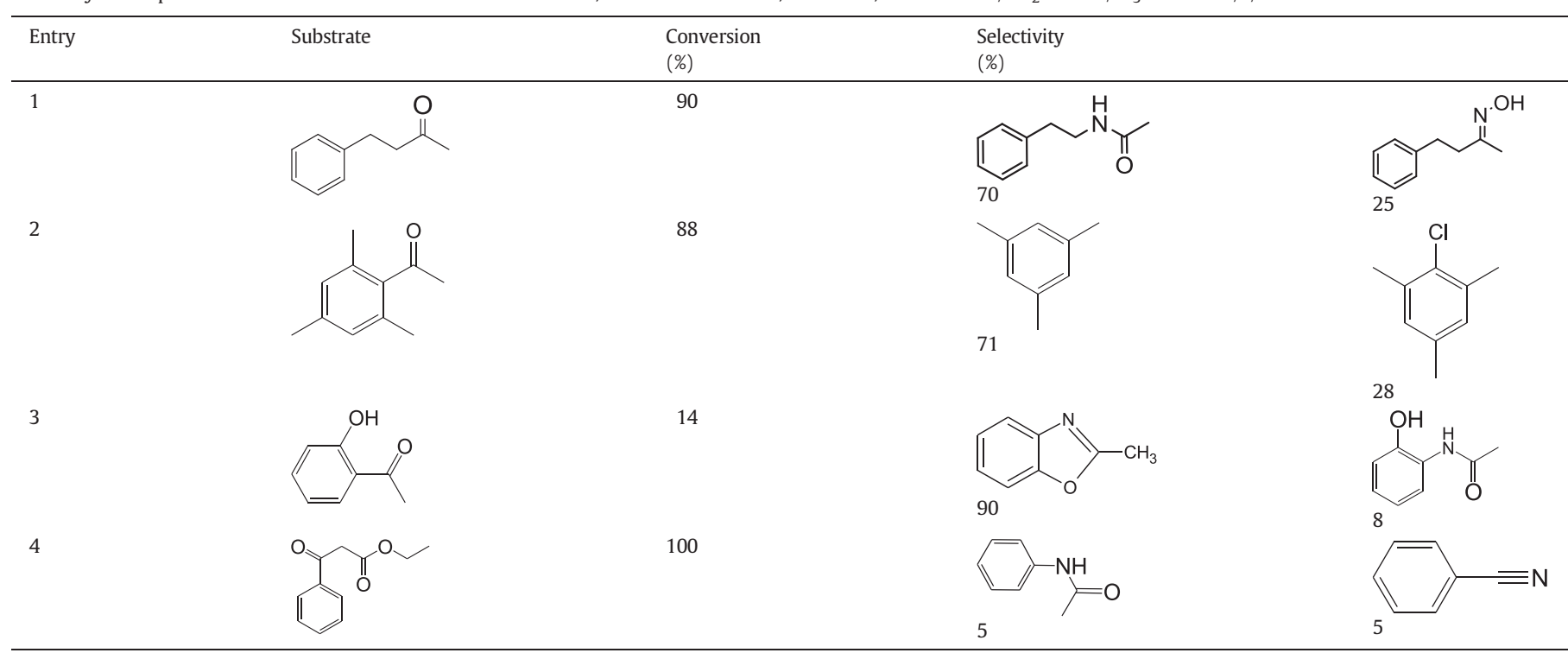


Table 3

Reactivity of some aldehydes. Run conditions: reaction time $=16 \mathrm{~h}$, substrate $=2 \mathrm{mmol}, \mathrm{T}=70{ }^{\circ} \mathrm{C}$, and substrate $/ \mathrm{NH}_{2} \mathrm{OH} \cdot \mathrm{HCl} / \mathrm{CF}_{3} \mathrm{COOH}=1 / 3 / 15$.

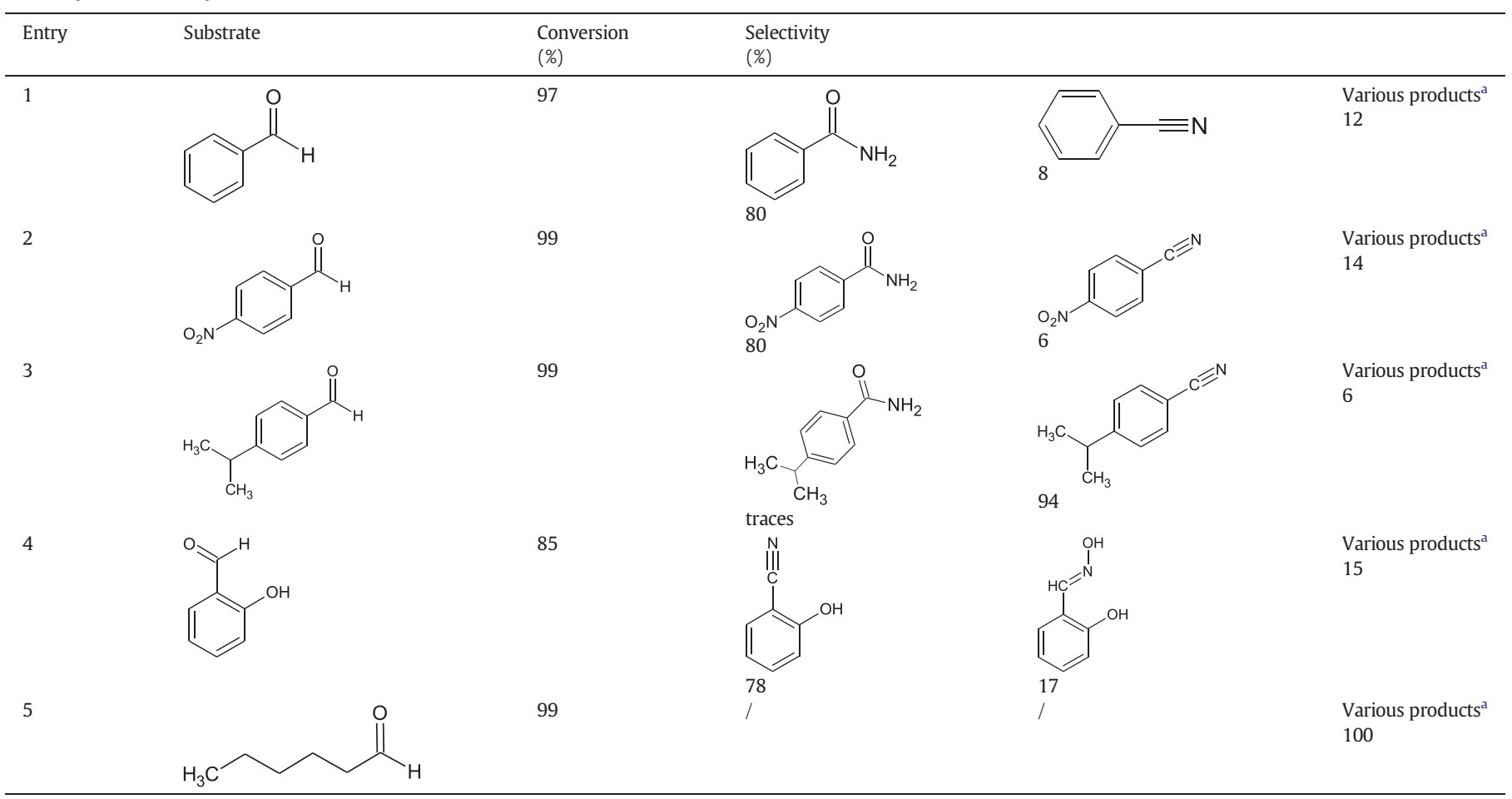

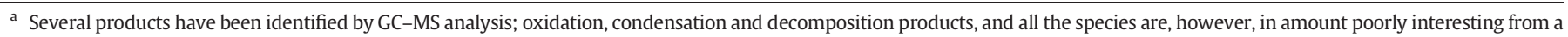
synthetic point of view.

hydroxylamine attack then the oximation reaction is the rate limiting step. Similar reactivity was also observed for aldehydes but the easy transformation to nitriles diminishes the intrinsic selectivity of the methods. The role of TFA in the reaction mechanism is under investigation, but most probably this reagent is actively involved in formation of the oxime ester compounds, according to a mechanism similar to the one reported in Scheme 1 for the Beckmann rearrangement of cyclohexanone oxime to $\varepsilon$-caprolactam.

\section{Acknowledgments}

Financial support by Ca' Foscari University of Venice is gratefully acknowledged (ADIR fund 2011). A special thank to Mr. Claudio Tortato for the helpful discussions.

\section{Appendix A. Supplementary data}

Supplementary data to this article can be found online at http://dx. doi.org/10.1016/j.catcom.2014.02.007.

\section{References}

[1] R. Opsahl, 4th ed., Kirk Othmer Encyclopaedia of Chemical Technology, vol. 2, J. Wiley, 1999.

[2] A. Aguilo, C. Hobbs, E.G. Zey, Ulmann's Encyclopaedia of Industrial Chemistry, 6th ed., J. Wiley, 1998.

[3] R.A. Nugent, C.H. Hall, 4th ed., Kirk Othmer Encyclopedia of Chemical Technology, vol. 2, J. Wiley, 1999 ,

[4] S.C. Mitchel, R.H. Waring, Ulmann Encyclopaedia of Industrial Chemistry, 6th ed. J. Wiley, 1998.

[5] T. Horlenko, J.R. Fritch, O.S. Fruchey, Hoechst Celanese Corporation, US Pat. 4954652, 1990.
[6] Y. Ogata, M. Okano, K. Matsumoto, J. Am. Chem. Soc. 77 (1955) 4643-4646.

[7] F. Greer, D.E. Pearson, J. Am. Chem. Soc. 77 (1955) 6649-6650.

[8] P.J. McNulty, D.E. Pearson, J. Am. Chem. Soc. 81 (1959) 612-618.

[9] M.I. Vinnik, N.G. Zarakhani, Russ. Chem. Rev. (Eng. Trans.) 36 (1967) 51-64.

[10] B.J. Gregory, R.B. Moodie, K. Schofield, J. Chem. Soc. B (1970) 338-346.

[11] S.G. Kim, T. Kawakami, T. Ando, Y. Yukawa, Bull. Chem. Soc. Jpn. 52 (1979) $1115-1120$.

[12] G. Petrini, G. Leonfanti, M.A. Mantegazza, F. Pignataro, in: P.T. Anastas, T.C. Williamson (Eds.), Green Chemistry, American Chemical Society, 1996, pp. 33-48.

[13] S. Tonti, P. Roffia, V. Gervasutti, to Enichem Anic S.p.A. EP, 0496385A1, 1992.

[14] J. Roeseler, W. Hoelderich, D. Arntz, to Degussa AG. DE, 19608 660.4, 1996.

[15] W.F. Holderich, G. Dahloff, H. Ichihashi, K. Sugita, to Sumitomo Chemical Company US Pat. 6,531,595 B2, 2003.

[16] G. Dahlhoff, J.P.M. Niederer, W.F. Hölderich, Catal. Rev. Sci. Eng. 43 (2001) 381-441.

[17] T. Okubo, S. Suzuki, T. Matsushita, T. Suzuki, to Sumitomo Chemical Co. EP 2157080 , 2009.

[18] X. Liu, L. Xiao, H. Wua, Z. Li, J. Chen, C. Xia, Catal. Commun. 10 (2009) 424-427.

[19] A. Zicmanis, S. Katkevica, P. Mekss, Catal. Commun. 10 (2009) 614-619.

[20] R. Mokaya, M. Poliakoff, Nature 437 (2005) 1243-1244.

[21] J.M. Thomas, R. Raja, Proc. Natl. Acad. Sci. 102 (2005) 13732-13736,

[22] W.F. Hoelderich, A. Mettu, R. Schaller, to Uhde/Inventa/Fischer EP 2098506, 2008

[23] A. Martínez-Asencio, M. Yus, D.J. Ramón, Tetrahedron 68 (2012) 3948-3951.

[24] M. Ashif Ali, T. Punniyamurthy, Adv. Synth. Catal. 352 (2010) 288-292.

[25] S. His, C. Meyer, J. Cossy, G. Emeric, A. Greine, Tetrahedron Lett. 44 (2003) 8581-8584.

[26] L. Ronchin, A. Vavasori, M. Bortoluzzi, Catal. Commun. 10 (2008) 251-256.

[27] L. Ronchin, M. Bortoluzzi, A. Vavasori, J. Mol. Struct.: Theochem 858 (2008) 46-51.

[28] L. Ronchin, A. Vavasori, J. Mol. Catal. A Chem. 313 (2009) 22-30.

[29] E. Rancan, G. Quartarone, L. Ronchin, A. Vavasori, Appl. Catal. A Gen. 472 (2014) $167-177$.

[30] J. S. Zhang, A. Riaud, K. Wang, Y. C. Lu, G. S. Luo, Catal. Lett. http://dx.doi.org/10.1007/ s10562-013-1114-3.

[31] J.S. Zhang, Y.C. Lu, K. Wang, G.S. Luo, Ind. Eng. Chem. Res. 52 (2013) 6377-6381.

[32] N.C. Marziano, L. Ronchin, C. Tortato, O. Tonon, R. Bertani, Int. J. Chem. Kinet. 36 (2004) 417-426.

[33] M.J. Craft, B.F. Landrum, E. Suratt, C.T. Lester, J. Am. Chem. Soc. 73 (1951) 4462-4463.

[34] N.C. Marziano, L. Ronchin, C. Tortato, Catal. Lett. 64 (2000) 15-22.

[35] E.C. Horning, V.L. Stromberg, H.A. Lloyd, J. Am. Chem. Soc. 74 (1952) 5153-5155. 